\title{
Bigger brains may make better problem-solving carnivores
}

\author{
Jennifer Vonk ${ }^{1}$ \\ Published online: 11 April 2016 \\ (C) Psychonomic Society, Inc. 2016
}

Summary Benson-Amram, Dantzer, Stricker, Swanson, \& Holekamp's (Proceedings of the National Academy of Sciences, 113, 25321-25376, 2016) recent demonstration that larger-brained carnivores were more successful in a single problem-solving task, relative to smaller-brained carnivores, irrespective of social complexity, poses a challenge to proponents of the social intelligence hypothesis (Humphrey, 1976) and provides some support for the idea that larger relative brain sizes have evolved to support greater problem-solving abilities. However, an important question, neglected by the authors, is the extent to which foraging ecology, rather than social environment, more accurately predicts problem solving, and whether this relationship would be observed in noncarnivore, noncaptive animals across a range of tasks.

Keywords Problem solving · Carnivores · Brain size · Foraging $\cdot$ Social intelligence

Benson-Amram and colleagues (Benson-Amram, Dantzer, Stricker, Swanson, \& Holekamp, 2016) recently published the results of an ambitious study designed to investigate whether problem-solving abilities in a large number of carnivore species (140 members of 39 species from nine families) could be predicted by neuroanatomical and socio-ecological measures. This undertaking is all the more impressive when one considers how grossly understudied carnivores have been with regard to their cognitive abilities, relative to other orders (e.g., primates, cetaceans, Passeriformes). This historical oversight is even more glaring when one considers the diversity of foraging ecologies, social structures, and brain sizes in the Carnivore order. The results of the study, based on a single problem-solving task, are intriguing in large part because they run counter to the oft-touted social intelligence hypothesis (Humphrey, 1976): Social complexity did not predict problem-solving success in this study, even though it had recently been shown to do so for a smaller number of species

Jennifer Vonk

vonk@oakland.edu

1 Department of Psychology, Oakland University, Rochester, MI, USA within the same order using a similar task (Borrego \& Gaines, 2016). One possible explanation for these differing conclusions may rest upon the dichotomous measure of success in the Benson-Amram et al. study, as compared to the more inclusive measure of innovation in Borrego and Gaines. Furthermore, the latter study was limited to three species of felines and hyenasspecies that do not vary considerably with regard to diet and brain size, perhaps leaving sociality as the only differentiating factor to explain differences in problem-solving ability. Neither study tested social problem solving, so both are mute with regard to the advantages of predictive factors for specific cognitive domains.

Because the numbers of individuals tested varied substantially for different species in Benson-Amram et al. (2016; from one to nine), it is difficult to draw firm conclusions regarding species differences, given the lack of attention to individual differences within a species, particularly in light of the vast differences in rearing history and environmental enrichment that captive animals experience. Furthermore, the number of individuals that eventually succeeded (a dichotomous measure of success) fails to capture the full range of cognitive traits involved in problem solving. Latency to solve the task, number of solutions attempted, persistence at the task, and so on, might be better measures of problem-solving ability than overall success, but these measures were used as predictors rather than outcomes. Motivation and comfort interacting with the apparatus are other factors that undoubtedly impact performance that were not considered in relation to species' success in the Benson-Amram study, although factors such as latency to approach and time spent trying to open the box, as well as dexterity, were included in their model. The authors did control for latency to approach the box, which could be considered a measure of motivation and/ or neophobia. However, an additional problem is that the number of trials presented to individuals ranged from three to ten. Although the authors conducted additional analyses limiting the sample to those with a certain minimum number of trials or minimum number of individuals tested, and found no difference in the pattern of results, this approach does not satisfy the concern that species are misrepresented when underrepresented.

Furthermore, as the authors acknowledged, it is challenging to design analogous tasks for diverse species that are specifically 
adapted for unique environments. Sometimes creating identical tasks introduces confounds, as animals differ in their dexterity, perceptual systems, neophobia, motivation, and so on. Tasks must be adapted to pose the same questions in similar ways to animals that differ on so many dimensions, introducing concerns over the comparability of the tasks. Benson-Amram and colleagues (2016) elected to use a steel mesh puzzle box that subjects had to open to retrieve a food reward. The authors indicated that the puzzle boxes were scaled for the animals' body sizes, but only two sizes of boxes were used, which suggests that the ratio of the box to the animals' body size was quite variable. The box could also be opened just by moving it, suggesting an advantage for larger, stronger, more persistent animals. It would be important to compare the trials solved by sliding the bolt versus the trials solved by persistent moving of the box. In watching the supplemental video, the box appears to open rather easily. I wonder whether the same results would be obtained with a more challenging puzzle — one requiring causal reasoning, for example.

The authors tackled a timely and controversial question regarding the association between relative (rather than absolute) brain size and cognitive complexity. One of the reasons this question is controversial is because of the difficulty defining what constitutes "advanced" cognition. Although, admittedly, problem solving could be a good measure of advanced cognition, if one thinks of problem solving as a generalized ability to quickly solve novel problems in a variety of contexts, here the authors presented only a single task, which involved the removal of a food reward from a puzzle box. The task might be considered most aligned with problems of extractive foraging, and therefore might be predicted by species' natural foraging ecologies. The technical intelligence hypothesis posits that dietary factors, such as the breadth, spread, unpredictability, and inaccessibility of diet, may be the best predictors of cognitive abilities such as problem solving and causal reasoning (Byrne, 1997). Given the untested buffer hypothesis (Sol, 2009), and the fact that the authors did not examine dietary factors, such as breadth of diet or the need for extractive foraging, in predicting success on what was essentially a foraging task, these unresolved questions may prompt future research.

Researchers must be careful to differentiate relative from absolute brain size, given that brain size is highly correlated with body size. Animals with larger-than-expected brain sizes for their body size pose an evolutionary dilemma: Because brain matter is expensive to produce, there must be benefits to offset these costs. However, suggesting that the obvious benefit is to increase cognitive complexity is simplistic, because large portions of the brain may be devoted to other functions, such as temperature regulation, circadian rhythms, perceptual processing, motor control, and various other functions that do not reflect our intuitions of cognitive complexity. Thus, it is important to focus on the sizes of specific brain areas, such as the prefrontal cortex or the frontal lobes, rather than focusing on overall brain size. Such complications may help explain the rather mixed data derived from studies attempting to demonstrate correlations between brain size and cognitive complexity. Importantly, in their study, BensonAmram et al. (2016) examined deviance information criterion model selection analyses to evaluate the relationship between four gross brain areas (cerebrum, posterior cerebrum, anterior cerebrum, and hindbrain), and the inclusion of these specific brain areas increased the model fit; however, these data were available for only a subset of the species tested.

Although group size has been shown to be an adequate proxy for social complexity, I would argue that complexity in social interactions can occur even in relatively solitary species that must compete at feeding sites or that spend significant time teaching offspring how to survive - something that might be true in bears, which performed well. Furthermore, the authors apparently did not consider whether the subjects lived in social groups in their captive environments, despite their potential designation as solitary animals. Although the social intelligence hypothesis clearly focuses on the natural lifestyles of members of a species in shaping the cognitive evolution of the species, it is possible that changes could occur during ontogeny if individuals are reared in social groups. It is also possible that exposure to human artifacts in a captive environment may impact cognitive abilities that might not emerge in wild environments. Therefore, some caution is warranted with any tests conducted in purely captive environments.

As someone who studies the cognitive abilities of bears, I am not surprised that they outperformed the other groups tested, despite their designation as solitary animals. BensonAmram and colleagues' (2016) study adds weight to the idea that foraging requirements may more strongly impact the evolution of intelligence. However, it is possible that the factors that are important in carnivores are not the same as those that drive intelligence in other species, such as corvids, primates, and cetaceans, so we must be careful not to generalize beyond carnivores until similar work is conducted with other species.

\section{References}

Benson-Amram, S., Dantzer, B., Stricker, G., Swanson, E. M., \& Holekamp, K. E. (2016). Brain size predicts problem-solving abilities in mammalian carnivores. Proceedings of the National Academy of Sciences, 113, 2532-2537. doi:10.1073/pnas. 1505913113

Borrego, N., \& Gaines, M. (2016). Social carnivores outperform asocial carnivores on an innovative problem. Animal Behaviour, 114, 21-26. doi:10.1016/j.anbehav.2016.01.013

Byrne, R. W. (1997). The Technical Intelligence hypothesis: An additional evolutionary stimulus to intelligence? In A. Whiten \& R. W. Byrne (Eds.), Machiavellian intelligence: Vol. II. Extensions and evaluations (pp. 289-311). Cambridge, UK: Cambridge University Press.

Humphrey, N. (1976). The social function of intellect. In P. P. G. Bateson \& R. A. Hinde (Eds.), Growing points in ethology (pp. 303-317). Cambridge, UK: Cambridge University Press.

Sol, D. (2009). The cognitive-buffer hypothesis for the evolution of large brains. In R. Dukas \& R. M. Ratcliffe (Eds.), Cognitive ecology II (pp. 111-136). Chicago, IL: University of Chicago Press. 